\title{
Response priming driven by local contrast, not subjective brightness
}

\author{
ThOMAS SCHMIDT \\ University of Kaiserslautern, Kaiserslautern, Germany \\ Sandra Miksch, Lisa Bulganin, Florian Jäger, and Felix Lossin \\ University of Giessen, Giessen, Germany \\ AND \\ Joline Jochum And Peter KoHL \\ University of Kaiserslautern, Kaiserslautern, Germany
}

\begin{abstract}
We demonstrate qualitative dissociations of brightness processing in visuomotor priming and conscious vision. Speeded keypress responses to the brighter of two luminance targets were performed in the presence of preceding dark and bright primes (clearly visible and flanking the targets) whose apparent brightness values were enhanced or attenuated by a visual illusion. Response times to the targets were greatly affected by consistent versus inconsistent arrangements of the primes, relative to the targets (response priming). Priming effects could systematically contradict subjective brightness matches, such that one prime could appear brighter than the other but could prime as if it were darker. Systematic variation of the illusion showed that response-priming effects depended only on local flanker-background contrast, not on the subjective appearance of the flankers. Our findings suggest that speeded motor responses, as opposed to conscious perceptual judgments, access an early phase of lightness and brightness processing prior to full lightness constancy.
\end{abstract}

The subjective perception of lightness and brightness is the final outcome of a complex processing system that tries to infer the physical properties of surfaces in the environment from the distribution of light on the retina. Both classic (Land \& McCann, 1971) and current (Adelson, 2000; Gilchrist et al., 1999) models of lightness perception stress that this is a multistage process: A 2-D pattern of raw local luminance or contrast values on the retina must be interpreted to disentangle 3-D surface properties from a bewildering array of lighting effects, such as illumination gradients, shadows, transparency, and gloss. This system's most basic task is to achieve lightness constancy by telling differences in reflectance (the percentage of light a surface reflects into the observer's eye) from differences in illumination (the amount of light incident on that surface; Adelson, 1993; Li \& Gilchrist, 1999). The term lightness is defined as the subjectively perceived reflectance of a surface, whereas the term brightness refers to its perceived luminance. For example, if you take a book from a sunny to a shadowy place, the coloring of the paper does not seem to change from white to gray, even though its luminance is vastly decreased and the pages appear less bright. Instead, the coloring of the pages remains largely invariant- that is, constant in lightness - corresponding more closely to its reflectance than to its luminance value (see Gilchrist, 2006, for further examples of lightness constancy).
Psychophysical judgments of lightness and brightness are typically based on the final outcome of the processing system - presumably, a highly integrated representation of surfaces and illuminants in visual awareness. But is it possible to access earlier, more preliminary steps in the process? In this article, we utilize the fact that visual stimuli are able to directly trigger assigned motor responses without mediation by visual awareness (Neumann, 1990). In the response priming paradigm (Neumann \& Klotz, 1994; Vorberg, Mattler, Heinecke, Schmidt, \& Schwarzbach, 2003), participants have to perform a speeded response to a target stimulus that is preceded by a prime stimulus triggering either the same response as the target (consistent prime) or the opposite response (inconsistent prime). Consistent primes speed responses to the target, whereas inconsistent primes slow them, and this priming effect increases with the stimulus onset asynchrony (SOA) between the prime and target (Vorberg et al., 2003). Strikingly, this typical time course of response priming is independent of the visibility of the prime: For SOAs up to $100 \mathrm{msec}$, it remains invariant under various conditions of visual masking, no matter whether the prime is identified perfectly or not at all, and no matter whether prime identification performance increases or decreases with SOA. In particular, priming effects can become larger with SOA even though the prime is becoming more difficult to

T. Schmidt, thomas.schmidt@sowi.uni-kl.de 
discriminate (Mattler, 2003b; Schmidt \& Vorberg, 2006; Vorberg et al., 2003).

Many studies have shown that primes in responsepriming tasks rapidly trigger the specific motor responses assigned to them, as measured in lateralized readiness potentials in the EEG (Dehaene et al., 1998; Eimer \& Schlaghecken, 1998, 2003; Jaśkowski, van der Lubbe, Schlotterbeck, \& Verleger, 2002; Leuthold \& Kopp, 1998; Vath \& Schmidt, 2007). Analyses of primed pointing responses to color stimuli indicate that it is the prime, rather than the actual target, that determines the onset of pointing, as well as the initial pointing direction (Schmidt, 2002). Importantly, the earliest phases of the priming effect depend only on prime, but not on target, characteristics, and they are invariant over widely differing degrees of masking (Schmidt, Niehaus, \& Nagel, 2006; Vath $\&$ Schmidt, 2007). The rapid-chase theory of response priming (Schmidt et al., 2006; Schmidt \& Schmidt, 2009) therefore proposes that response priming is based on sequential, nonoverlapping waves of rapid feedforward activation (feedforward sweeps; Lamme \& Roelfsema, 2000; see also Bullier, 2001; VanRullen \& Thorpe, 2002), which are elicited in turn by primes and targets and traverse the visuomotor system in strict sequence, directly triggering the assigned responses (Neumann, 1990). Feedforward activation by the primes would still be largely free of feedback information; it would thus outrun the recurrent processing often assumed necessary for generating visual awareness of the primes (Di Lollo, Enns, \& Rensink, 2000; Lamme \& Roelfsema, 2000). This idea not only would explain the well-established independence of priming and prime awareness, but also would suggest that the earliest signs of motor output might still depend on a stimulus signal that is, in some respects, less elaborate than output generated after fully recurrent processing.

In this article, we want to examine whether responsepriming effects can be used to access early, preconscious stages of brightness processing by picking up the earliest processing sweeps engaging the motor system. We employed a stimulus similar to the corrugated plaid illusion introduced by Adelson (1993) and used a variant of response priming with unmasked flanker primes (Eriksen \& Eriksen, 1974; Mattler, 2003a). In our stimulus (Figure 1), one high-luminance and one low-luminance target square appeared on a folded plane of intermediate luminance. The plane was seemingly illuminated from the side, so that different segments were lighted, shadowed, or illuminated neutrally. Participants performed speeded responses indicating the location of the brighter target. Targets were preceded by flanking stimuli serving as response primes. On consistent trials, each flanker had the same luminance as its adjacent target, whereas on inconsistent trials, flanker luminances were spatially switched with respect to target luminances. Consistent flankers were expected to speed responses to the targets, whereas inconsistent flankers were expected to slow responses.

Even though the flankers always had the same two luminance levels as the targets, their perceived brightness values depended on their placement on the different segments of the folded plane: If the high-luminance and low-

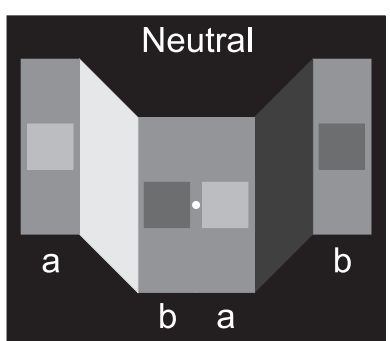

Target/flanker luminance:

$\mathrm{a}=20.9 \mathrm{~cd} / \mathrm{m}^{2}$

$\mathrm{b}=4.5 \mathrm{~cd} / \mathrm{m}^{2}$
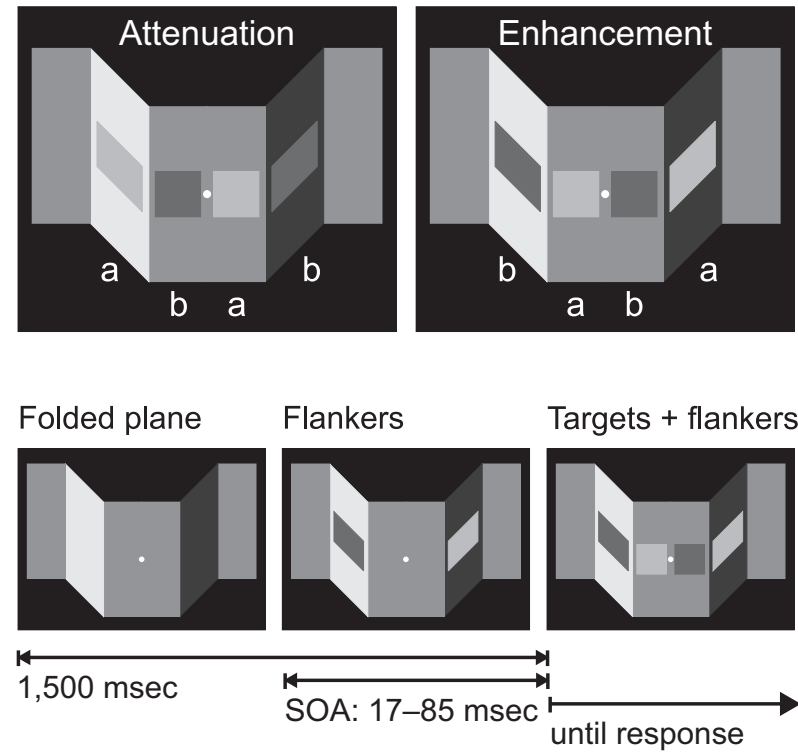

Figure 1. One high-luminance and one low-luminance target appeared in the central part of a folded plane seemingly illuminated from the side (schematic; see Table 1 for exact luminances). Flankers appeared on the outer, neutrally illuminated segments (neutral condition) or on the shadow and light segments. Depending on the illumination, the perceived brightness difference between the flankers could be attenuated or enhanced. Note that all targets and flankers labeled $a$ and $b$ have identical respective luminances, despite their different appearance. Only inconsistent trials are shown where flanker luminances are spatially switched with respect to target luminances. Lower panel: Participants first fixated on the folded plane, then responded by pressing a key on the side of the brighter target. Flankers appeared shortly before the targets. All the stimuli were clearly visible and remained on screen until the response was finished. SOA, stimulus onset asynchrony.

luminance flankers appeared on the lighted and shadowed segments, respectively, they seemed to be quite similar in brightness; if their placement on the lighted and shadowed segments was reversed, they seemed to be very dissimilar (Figure 1). According to Adelson (1993, 2000), this illusion is a feat of lightness constancy: The system is taking effects of illumination into account to arrive at estimates of the actual reflectances of the flankers, such that a flanker with constant luminance is judged lighter in surface color when lying in shadow than when lying in light and may also appear to be brighter - that is, to emit more light. Our major question was whether rapid motor activation elicited by the primes would respond to the illusion in a way consistent with subjective brightness judgments, or whether priming effects would in some way be dissociated from conscious brightness perception. 
Table 1

Luminances Employed in Experiments 1 and 2 (in $\mathrm{cd} / \mathrm{m}^{2}$ )

\begin{tabular}{|c|c|c|c|c|c|}
\hline $\begin{array}{c}\text { L:S } \\
\text { Ratio }\end{array}$ & $\begin{array}{l}\text { Lighted } \\
\text { Segment }\end{array}$ & $\begin{array}{c}\text { Shadowed } \\
\text { Segment }\end{array}$ & $\begin{array}{l}\text { Neutral } \\
\text { Segment }\end{array}$ & $\begin{array}{c}\text { High- } \\
\text { Luminance } \\
\text { Target or } \\
\text { Flanker }\end{array}$ & $\begin{array}{c}\text { Low- } \\
\text { Luminance } \\
\text { Target or } \\
\text { Flanker }\end{array}$ \\
\hline 336.26 & 43.86 & 0.13 & 11.10 & 20.89 & 4.53 \\
\hline 86.73 & 38.85 & 0.45 & 11.10 & 20.89 & 4.53 \\
\hline $34.75^{*}$ & $34.17^{*}$ & $0.98^{*}$ & $11.10^{*}$ & $20.89^{*}$ & $4.53^{*}$ \\
\hline 13.77 & 28.43 & 2.06 & 11.10 & 20.89 & 4.53 \\
\hline 7.74 & 24.50 & 3.17 & 11.10 & 20.89 & 4.53 \\
\hline 3.34 & 18.66 & 5.59 & 11.10 & 20.89 & 4.53 \\
\hline 1.56 & 13.67 & 8.78 & 11.10 & 20.89 & 4.53 \\
\hline
\end{tabular}

Note-Light:shadow (L:S) ratio denotes the luminance ratio of lighted to shadowed segments. *Employed in Experiment 1.

\section{EXPERIMENT 1}

\section{Method}

Participants. Eight students from the University of Giessen (19-24 years of age, all female, 1 left-handed, all with normal or corrected-to-normal vision) participated for payment or course credit after giving informed consent.

Apparatus. The experiment was controlled by a $300-\mathrm{MHz}$ personal computer driving a 14 -in. VGA color monitor $(640 \times 480$ pixels) in synchrony with the monitor retrace rate of $60 \mathrm{~Hz}$. The monitor was viewed from a distance of approximately $1 \mathrm{~m}$ in a dimly lit room.

Stimuli and General Procedure. The brightness illusion was induced as shown schematically in Figure 1 (see Tables 1 and 2 for exact luminance and contrast values). The folded plane subtended $6.9^{\circ} \times$ $4.6^{\circ}$ of visual angle and appeared illuminated from the side such that the shadowed, lighted, and neutrally illuminated (frontoparallel) segments had luminances of $1.0,34.2$, and $11.1 \mathrm{~cd} / \mathrm{m}^{2}$, respectively. One high-luminance and one low-luminance target $\left(T_{\text {high }}\right.$ and $T_{\text {low }} ; 0.9^{\circ} \times$ $0.9^{\circ} ; 20.9$ and $4.5 \mathrm{~cd} / \mathrm{m}^{2}$, respectively) appeared side by side on the central frontoparallel segment of the plane. One high-luminance $\left(F_{\text {high }}\right)$ and one low-luminance $\left(F_{\text {low }}\right)$ flanker with luminances identical to those of $T_{\text {high }}$ and $T_{\text {low }}$ appeared either on the outer frontoparallel segments of the folded plane (neutral condition) or appropriately tilted on the shadowed and lighted segments. The illusion modulated the perceived brightness of the flankers: If $F_{\text {high }}$ and $F_{\text {low }}$ fell on the lighted and shadowed segments of the plane, respectively, their apparent difference in brightness was attenuated; if they fell on shadowed and lighted segments instead, their apparent difference was enhanced. On consistent trials, each flanker had the same luminance as its ad- jacent target, whereas on inconsistent trials, flanker luminances were spatially switched with respect to target luminances.

At the beginning of each trial, the folded plane appeared against a dark screen $\left(0.01 \mathrm{~cd} / \mathrm{m}^{2}\right)$, with a white fixation point $\left(63.0 \mathrm{~cd} / \mathrm{m}^{2}\right)$ at the center of the midsegment. Following a variable fixation interval, the two flankers appeared either on the (neutrally lighted) outer segments or on the lighted and shadowed segments. Following a flankertarget SOA of $17,33,50,67$, or $83 \mathrm{msec}$, the two targets appeared to the left and right of the fixation point on the midsegment $1,500 \mathrm{msec}$ after the onset of the plane. All combinations of illusion direction (attenuation, neutral, or enhancement), illumination direction (left or right), target configuration ( $T_{\text {high }}$ left or right), and flanker-target consistency appeared equiprobably in pseudorandomized order. All the stimuli remained on screen until the participants had responded. The participants responded with their digit fingers on the " 1 " and " 3 " keys of the numerical keypad. They were instructed to keep their eyes on the fixation point and to respond as quickly and correctly as possible, pressing the key on the side where the brighter target was presented. Correct and incorrect responses triggered 100-msec feedback tones of 2000 or $200 \mathrm{~Hz}$, respectively. The next trial started after an intertrial interval of 1,000 msec. At the end of each block, the participants received feedback about their average response times (RTs) and percentages of correct responses. The participants took part in four 1-h sessions, each consisting of 20 blocks of 40 trials.

In a separate brightness-matching session, the participants used the computer keyboard to freely adjust the luminances of the targets to match the perceived luminance of the flankers. Note that the participants were instructed to match the brightness, not the lightness, of the flankers. Left targets were matched to left flankers, and right targets to right flankers. Flankers and targets appeared simultaneously in all the lighting conditions and remained on screen until both matches had been performed. Each participant performed 16 trials per stimulus condition. All the stimulus conditions appeared equiprobably in pseudorandomized order.

Data treatment and statistical methods. RT was defined as the time interval from target onset to registered keypress. Practice blocks and incorrect responses were excluded from analysis. RTs shorter than $100 \mathrm{msec}$ or longer than $800 \mathrm{msec}$ were discarded as outliers $(0.19 \%$ of the data). Repeated measures ANOVAs were performed with Greenhouse-Geisser-corrected $p$ values. Error rates were arcsine transformed to match ANOVA requirements.

\section{Results}

Priming effects were defined as RT differences in the consistent and inconsistent conditions $\left(\mathrm{RT}_{\text {incon }}-\mathrm{RT}_{\text {con }}\right)$. In the neutral and enhancement conditions, priming effects were positive $[F(1,7)=97.8$ and 157.2 , respectively;

Table 2

Log Local Flanker Contrast (Log Flanker-to-Background Luminance Ratio) in Experiment 2 Under Different Illumination Conditions

\begin{tabular}{cccccc}
\hline & \multicolumn{2}{c}{ Enhancement } & & \multicolumn{2}{c}{ Attenuation } \\
\cline { 2 - 3 } \cline { 5 - 6 } L:S & $\begin{array}{c}\text { Log Local } \\
\text { Contrast of } \\
\text { High-Luminance }\end{array}$ & $\begin{array}{c}\text { Log Local } \\
\text { Contrast of } \\
\text { Low-Luminance }\end{array}$ & & $\begin{array}{c}\text { Log Local } \\
\text { Contrast of } \\
\text { High-Luminance }\end{array}$ & $\begin{array}{c}\text { Log Local } \\
\text { Contrast of } \\
\text { Low-Luminance }\end{array}$ \\
\hline 336.26 & Flanker & Flanker & & Flanker & Flanker \\
86.73 & +5.08 & -2.27 & & $\mathbf{- 0 . 7 4}$ & $\mathbf{+ 3 . 5 5}$ \\
$34.75^{*}$ & +3.84 & -2.15 & & $\mathbf{- 0 . 6 2}$ & $\mathbf{+ 2 . 3 1}$ \\
13.77 & $+3.06^{*}$ & $-2.02^{*}$ & & $\mathbf{- 0 . 4 9 *}$ & $\mathbf{+ 1 . 5 3}$ \\
7.74 & +2.32 & -1.84 & & $\mathbf{- 0 . 3 1}$ & $\mathbf{+ 0 . 7 9}$ \\
3.34 & +1.89 & -1.69 & & $-\mathbf{0 . 1 6}$ & $\mathbf{+ 0 . 3 6}$ \\
1.56 & +1.32 & -1.42 & & +0.11 & -0.21 \\
\hline
\end{tabular}

Note-Under attenuation conditions, the sign of log local contrast reverses for light:shadow $(\mathrm{L}: \mathrm{S})$ ratios $\geq 7.74$ (bold print), indicating that the more luminant flanker becomes less luminant than the lighted background and the less luminant flanker becomes more luminant than the shadowed background. *Employed in Experiment 1. 
both $p$ s $<.0001]$, such that consistent flankers led to faster responses than did inconsistent flankers. Priming effects increased with increases in the flanker-target SOA in both conditions $[F(4,28)=16.3$ and 11.8 , respectively; both $p \mathrm{~s}<.0001$; see Figures 2 and 3, upper panels]. No main effects of SOA were observed. Priming effects were larger in the enhancement than in the neutral condition $[F(1,7)=$ $121.8, p<.0001]$ but did not increase more steeply with SOA $[F(4,28)=1.7, p=.1684]$. Surprisingly, priming effects were reversed in the attenuation condition: RTs on consistent trials were significantly longer than those on inconsistent trials, so that priming effects turned negative
$[F(1,7)=26.6, p=.0013]$. This reverse priming effect varied little across SOAs $[F(1,28)=1.2, p=.3310]$.

Similar effects were observed in the error rates (Figure 2, lower panel). In the neutral and enhancement conditions, errors occurred on trials on which flankers were inconsistent and the SOA was long enough to allow the prime to provoke the wrong response (Vorberg et al., 2003). Hardly any errors were observed on consistent trials. Priming effects (error incon $_{-}-$error $_{\text {con }}$ ) were positive in both conditions $[F(1,7)=3.7$ and $66.5, p=.0960$ and $p<.0001$, respectively], increased with SOA $[F(4,28)=$ 4.7 and $14.4, p=.0243$ and $p<.0001$, respectively], and
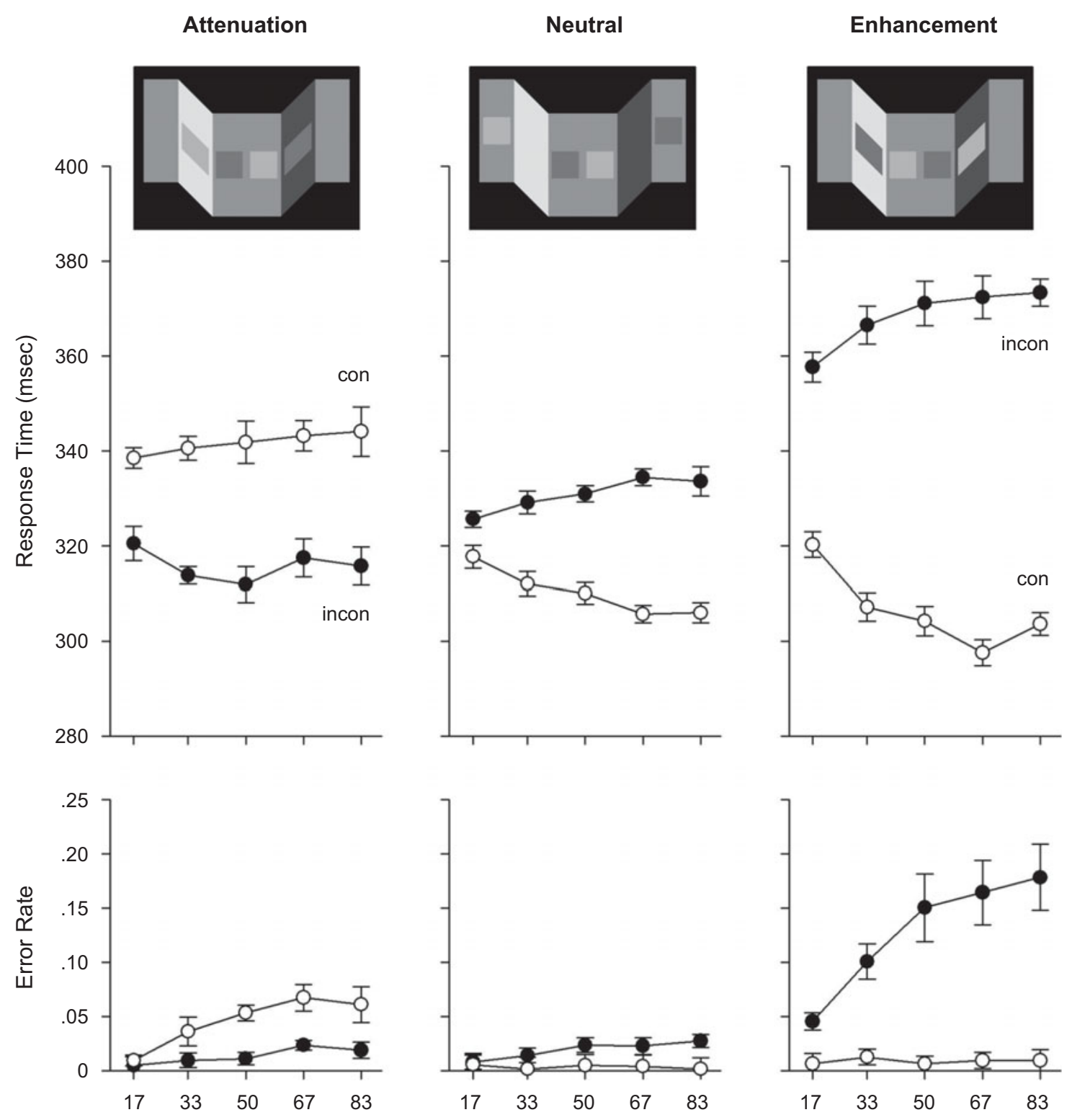

Flanker-Target SOA (msec)

Figure 2. Upper panel: Response times to the target stimuli in Experiment 1. Lower panel: Error rates. In all panels, the left, middle, and right columns show data from the attenuation, neutral, and enhancement conditions, respectively. All stimulus insets display consistent trials. SOA, stimulus onset asynchrony. Error bars represent standard errors of the means corrected for intersubject variance (Bakeman \& McArthur, 1996; cf. Loftus \& Masson, 1994). 

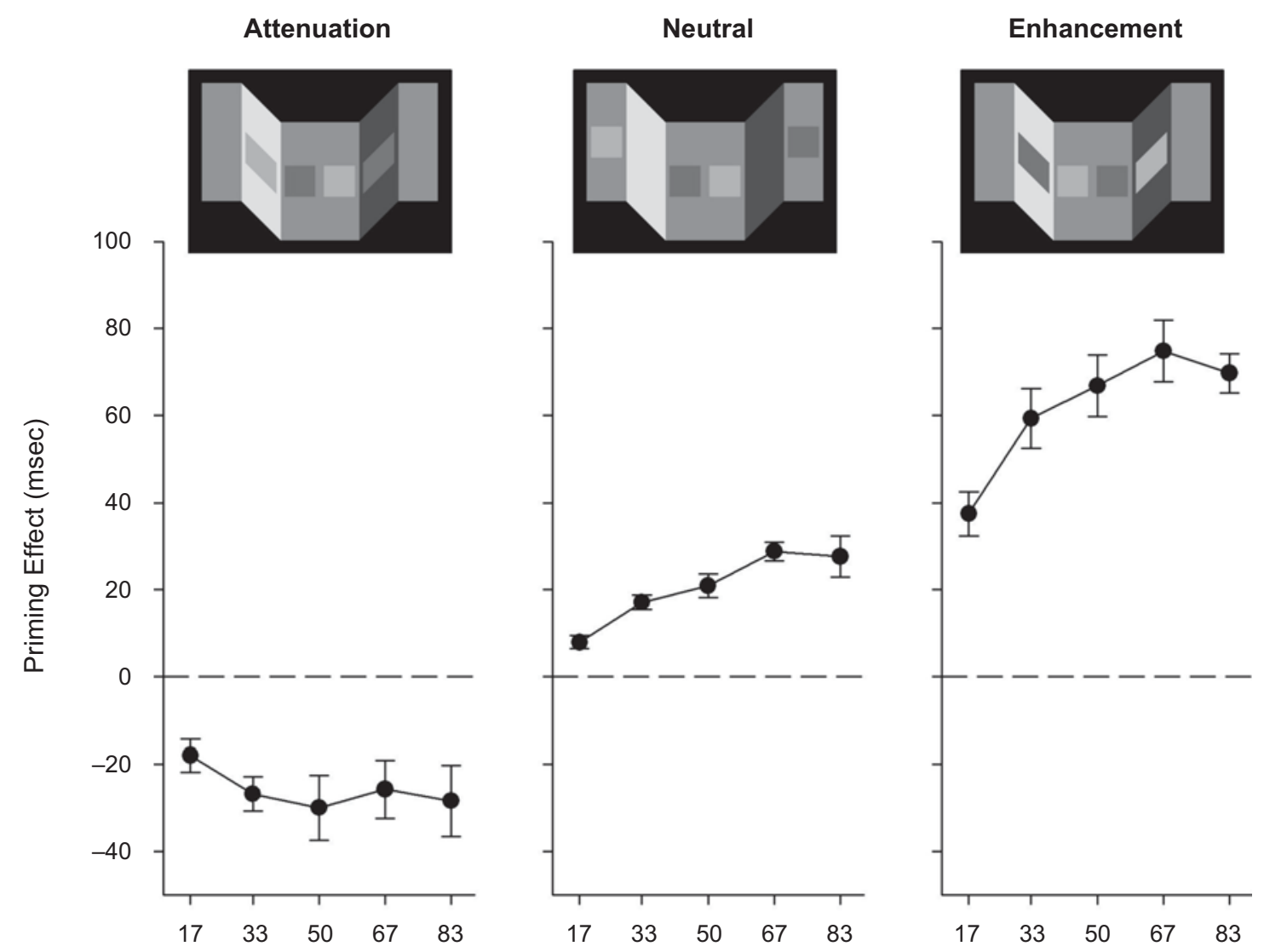

Flanker-Target SOA (msec)
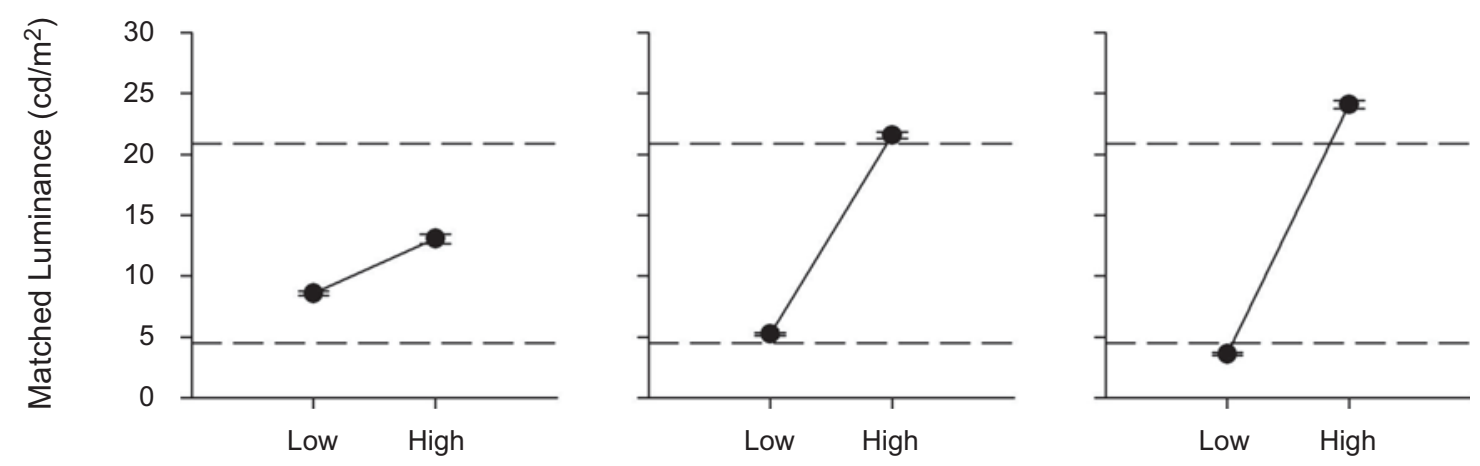

Flanker Luminance $\left(\mathrm{cd} / \mathrm{m}^{2}\right)$

Figure 3. Upper panel: Priming effects $\left(\mathbf{R T}_{\text {incon }}-\mathbf{R T _ { \text { con } }}\right)$ in responses to the targets in Experiment 1. Lower panel: Brightness matches of the target stimuli to the adjacent flanker stimuli indicate that the more luminant flanker always appeared brighter than the less luminant one. Stippled horizontal lines indicate the flankers' true luminances. SOA, stimulus onset asynchrony. Error bars represent standard errors of the means corrected for intersubject variance.

increased more steeply when flankers were inconsistent $[F(4,28)=4.6$ and $13.4, p=.0159$ and $p<.0001$, respectively]. In the enhancement condition, error rates were higher $[F(1,7)=57.4, p=.0001]$ and increased more quickly with SOA $[F(4,28)=2.7, p=.0633]$ than in the neutral condition. As for the RTs, the priming effect was reversed in the attenuation condition, with higher error rates now on consistent than on inconsistent trials $[F(1,7)=61.7, p=.0001]$. Error rates increased with
SOA $[F(4,28)=16.0, p=.0001]$, but the increase was not significantly different for consistent and inconsistent flankers.

Analysis of the brightness-matching task revealed a strong interaction of flanker luminance and illusion condition $[F(2,14)=275.9, p<.0001]$, confirming the perceptual effect of the illusion (Figure 3, lower panel). In the neutral condition, the participants were essentially veridical in matching the targets' luminances to those of 
the flankers. In the attenuation condition, high-luminance flankers were judged darker than in the neutral condition $[F(1,7)=199.6, p<.0001]$, whereas low-luminance flankers were judged brighter $[F(1,7)=130.4, p<$ $.0001]$. The reverse happened in the enhancement condition, where high-luminance flankers were judged brighter than in the neutral conditions $[F(1,7)=40.6, p=.0004]$, whereas low-luminance flankers were judged darker $[F(1,7)=137.1, p<.0001]$. The most crucial finding was that in all the illusion conditions, the more luminant flanker continued to appear brighter than the less luminant flanker despite the reversal in priming effects [attenuation condition, $F(1,7)=26.9, p=.0013$; neutral condition, $F(1,7)=854.7, p<.0001$; enhancement condition, $F(1,7)=581.2, p<.0001]$.

\section{Discussion}

The results from the neutral condition confirm clear effects of response priming: Consistent flankers speed responses to the targets, whereas inconsistent flankers slow responses, and this priming effect increases with flankertarget SOA (Vorberg et al., 2003). Error rates behave similarly, with errors occurring mainly on inconsistent trials at long SOAs. Priming effects differ strongly across illusion conditions. When the illumination of the folded plane enhances the perceived brightness difference of the flankers, priming effects increase sharply in RTs, as well as in error rates. But when the illumination attenuates perceived flanker differences, priming effects unexpectedly are reversed: Responses become slower with consistent flankers and faster with inconsistent flankers. This finding is opposite to the perceptual reports measured in the matching task, where the more luminant flanker is always judged brighter than the less luminant one, even under attenuation conditions. In other words, in the attenuation condition, the more luminant flanker looks brighter than the other flanker but primes as if it were darker.

How can this dissociation be explained? One explanation is in terms of local luminance contrasts of the flankers against the different backgrounds. In the neutral as well as the enhancement conditions, flankers appeared against an intermediate background, so that the more luminant of the two flankers was also a local luminance increment, whereas the less luminant flanker was a decrement. In the attenuation condition, however, the high-luminance flanker was a local decrement because it appeared on an even more luminant background, whereas the lowluminance flanker was a local increment because it appeared on an even darker background. In that condition, flanker-background contrast was reversed, as compared with flanker-flanker contrast (Table 2). The reversal in priming effects could be easily explained by assuming that response priming is driven by local contrast, not by perceived brightness.

Experiment 2 was performed to test the hypothesis that priming effects reverse because of the reversal in local flanker-to-background contrast. We employed the same stimuli as those in Experiment 1 but systematically varied the relative contrast of the light and shadow segments. In this way, we could exactly predict the stimulus conditions in which the flankers would turn from local increments into local decrements and vice versa, allowing us to test whether priming effects would be reversed under those exact conditions.

\section{EXPERIMENT 2}

\section{Method}

Participants. Eight students from the University of Giessen (2024 years of age, 3 male, 1 left-handed, all with normal or correctedto-normal vision) participated for payment or course credit after giving informed consent.

Stimuli and Procedure. The equipment and experimental task were identical to those in Experiment 1. Stimulus conditions were also identical, except that the luminances of the shadowed and lighted segments were varied in seven steps $\left(13.7-43.9 \mathrm{~cd} / \mathrm{m}^{2}\right.$ for lighted segments, $0.1-8.8 \mathrm{~cd} / \mathrm{m}^{2}$ for shadowed segments; light-shadow contrast between 1.6 and 336.3; see Tables 1 and 2 for details). Furthermore, we used only three flanker-target SOAs $(17,50$, and $83 \mathrm{msec})$ and dropped the neutral condition. The participants took part in eight 1-h sessions, each consisting of 20 blocks of 42 trials. In a separate brightness-matching session (with the procedure identical to that in Experiment 1), the flankers and targets appeared simultaneously in all the lighting conditions, and each participant performed 12 trials per stimulus condition. In all the sessions, stimulus conditions appeared equiprobably in pseudorandomized order.

Data treatment and statistical methods. Outlier detection in the priming task was performed as in Experiment 1. We discarded $0.90 \%$ of the data.

\section{Results}

In all the enhancement conditions, priming effects $\left(\mathrm{RT}_{\text {incon }}-\mathrm{RT}_{\text {con }}\right)$ were significantly positive, with faster responses to targets preceded by consistent flankers [all $F_{\mathrm{s}}(1,5) \geq 52.1$, all $\left.p \mathrm{~s} \leq .0008\right]$. Overall, priming effects increased with SOA $[F(2,10)=25.5, p=.0002]$ and were not significantly different across light-shadow levels (Figures 4 and 5). In the attenuation conditions, however, priming effects were reversed in exactly those conditions in which flanker-to-background contrast was reversed, such that the local flanker contrasts changed from increments to decrements (and vice versa). Specifically, priming effects varied strongly across contrast levels $[F(6,30)=21.6, p=.0012]$, starting out positive at low light-shadow contrast but becoming increasingly negative with increasing light-shadow contrast. There was an interaction of light-shadow level and SOA, indicating that the absolute amplitude of the priming effect (whether positive or negative) increased with SOA $[F(12,60)=12.9, p=$ $.0002]$. Priming effects were significantly positive for the two lowest light-shadow contrasts [both $F_{\mathrm{s}}(1,5) \geq 8.5$, both $p \mathrm{~s} \leq .0335]$ and were significantly negative for the five remaining light-shadow contrasts where local flanker contrast was reversed [all $F_{\mathrm{s}}(1,5) \geq 7.3$, all $p \mathrm{~s} \leq .0426$; see Figures 4 and 5].

Similar effects were observed in the error rates (Figure 4). In the enhancement conditions, error rates were higher for inconsistent than for consistent flankers $[F(1,5)=49.4, p=.0009]$, and this priming effect $\left(\right.$ error $_{\text {incon }}-$ error $\left._{\text {con }}\right)$ increased with SOA $[F(2,10)=$ $32.1, p<.0003$ ] similarly for the different light-shadow levels. In the attenuation conditions, priming effects in the error rates were reversed in the same conditions as 


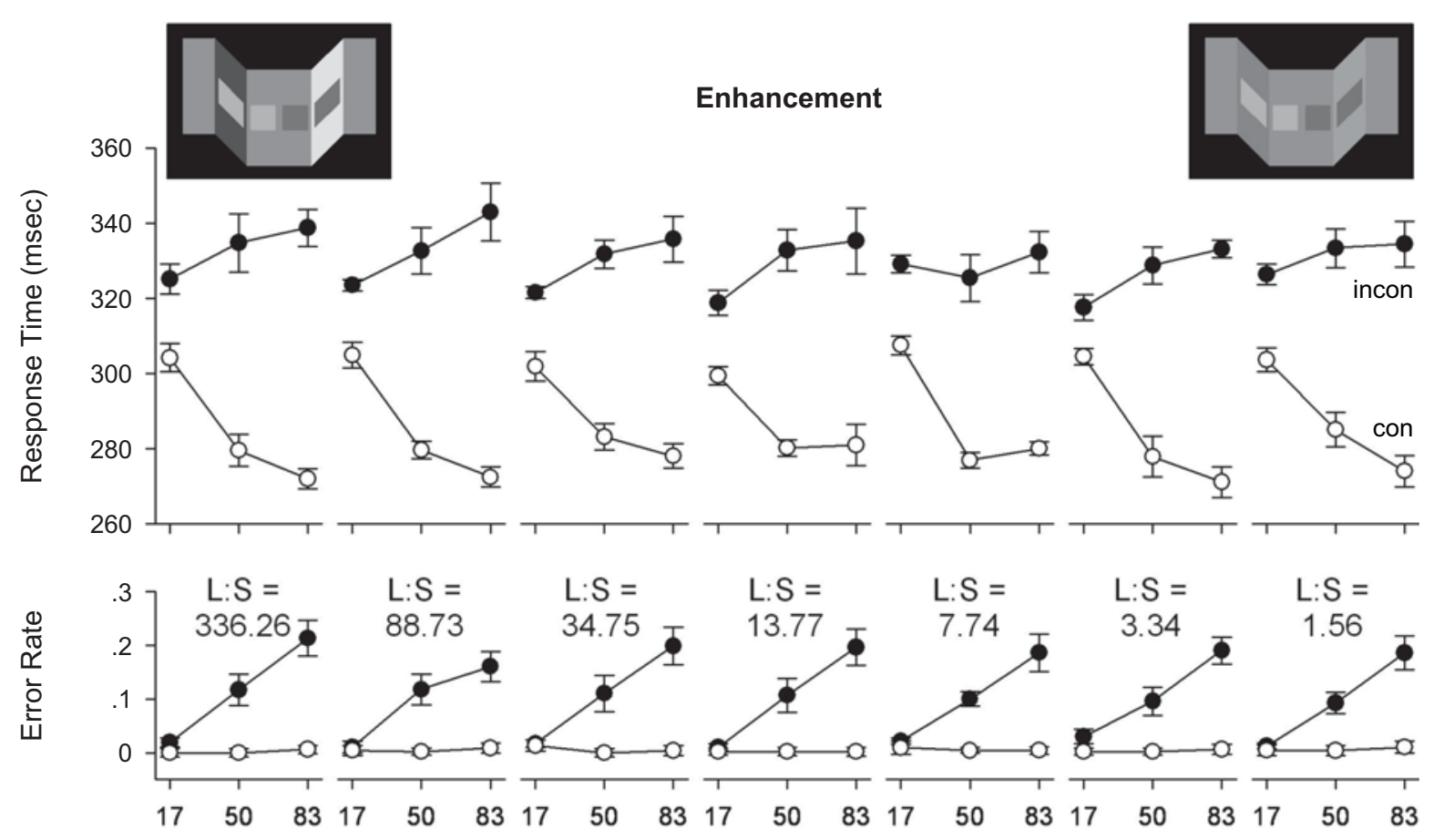

Flanker-Target SOA (msec)
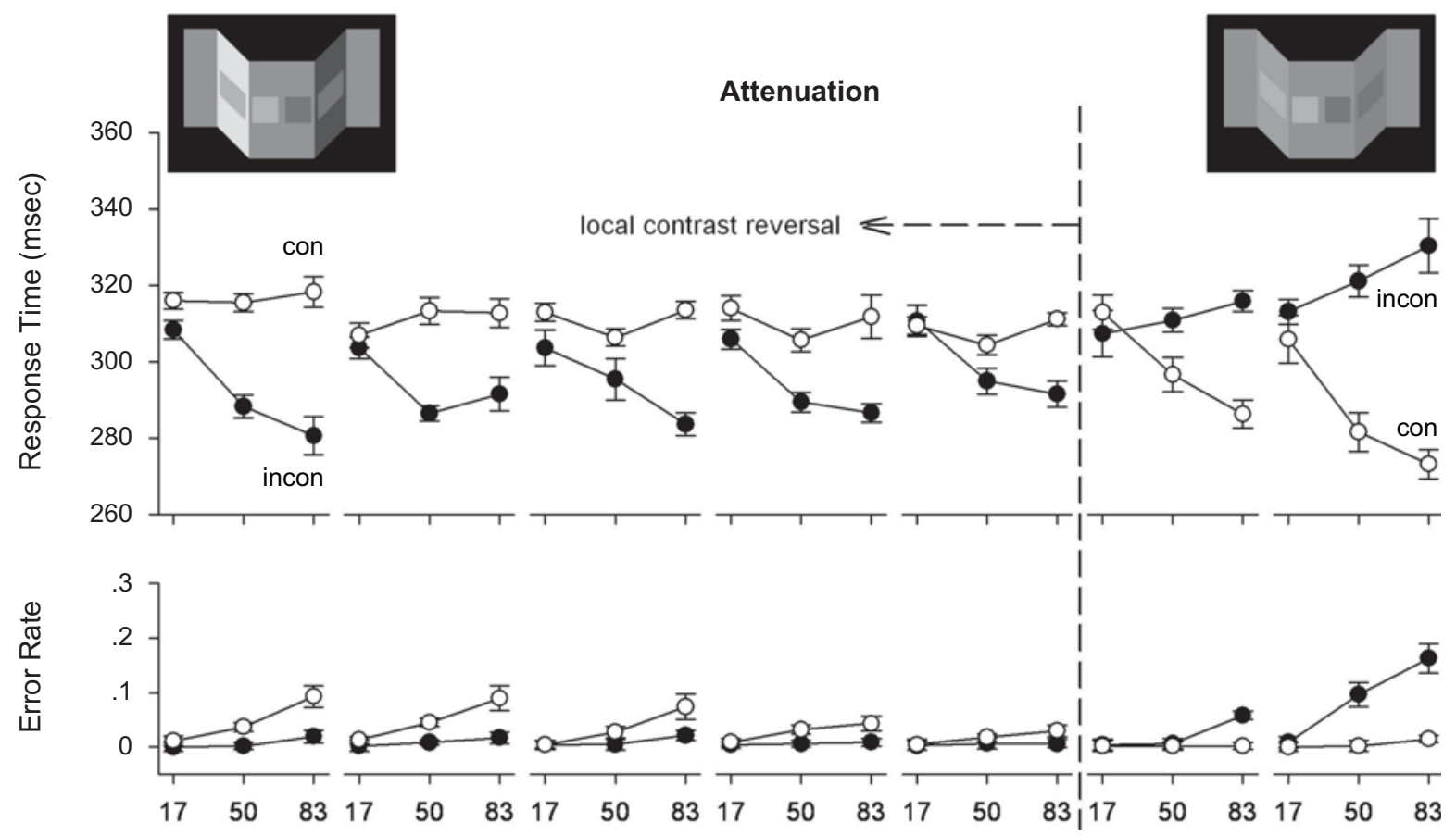

Flanker-Target SOA (msec)

Figure 4. Upper panels: Response times and error rates in the enhancement conditions in Experiment 2. Lower panels: Response times and error rates in the attenuation conditions. Light:shadow (L:S) contrast decreases from left to right. In the attenuation condition, the conditions are marked where local flanker contrast is reversed with respect to flanker-flanker contrast. SOA, stimulus onset asynchrony. Error bars represent standard errors of the means corrected for intersubject variance. 


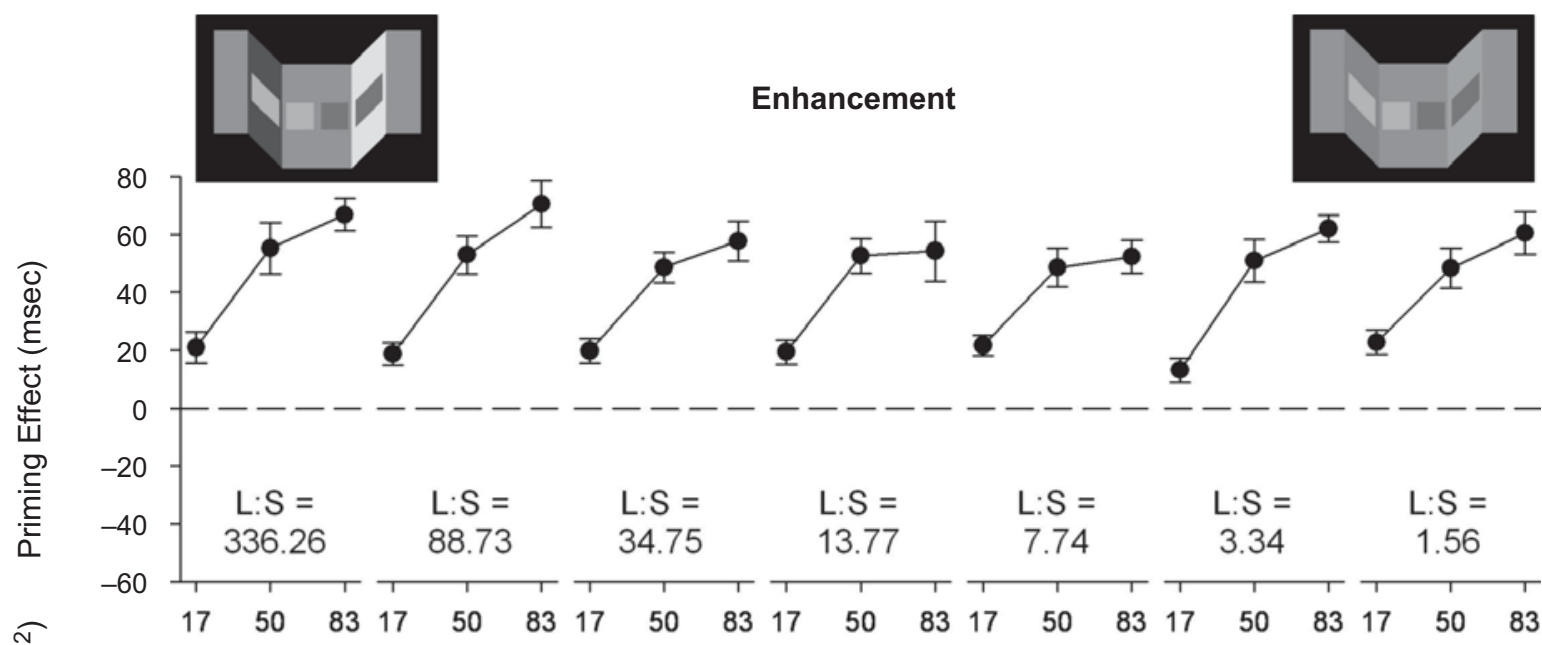

Flanker-Target SOA (msec)

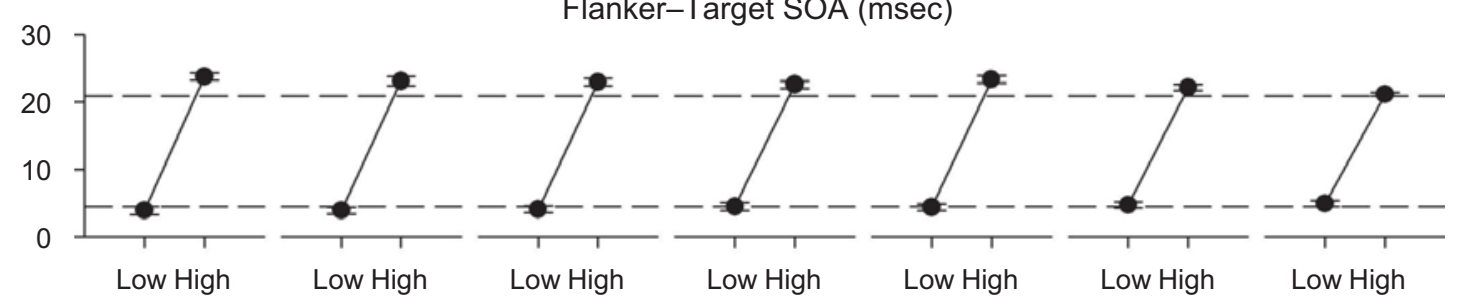

Flanker Luminance

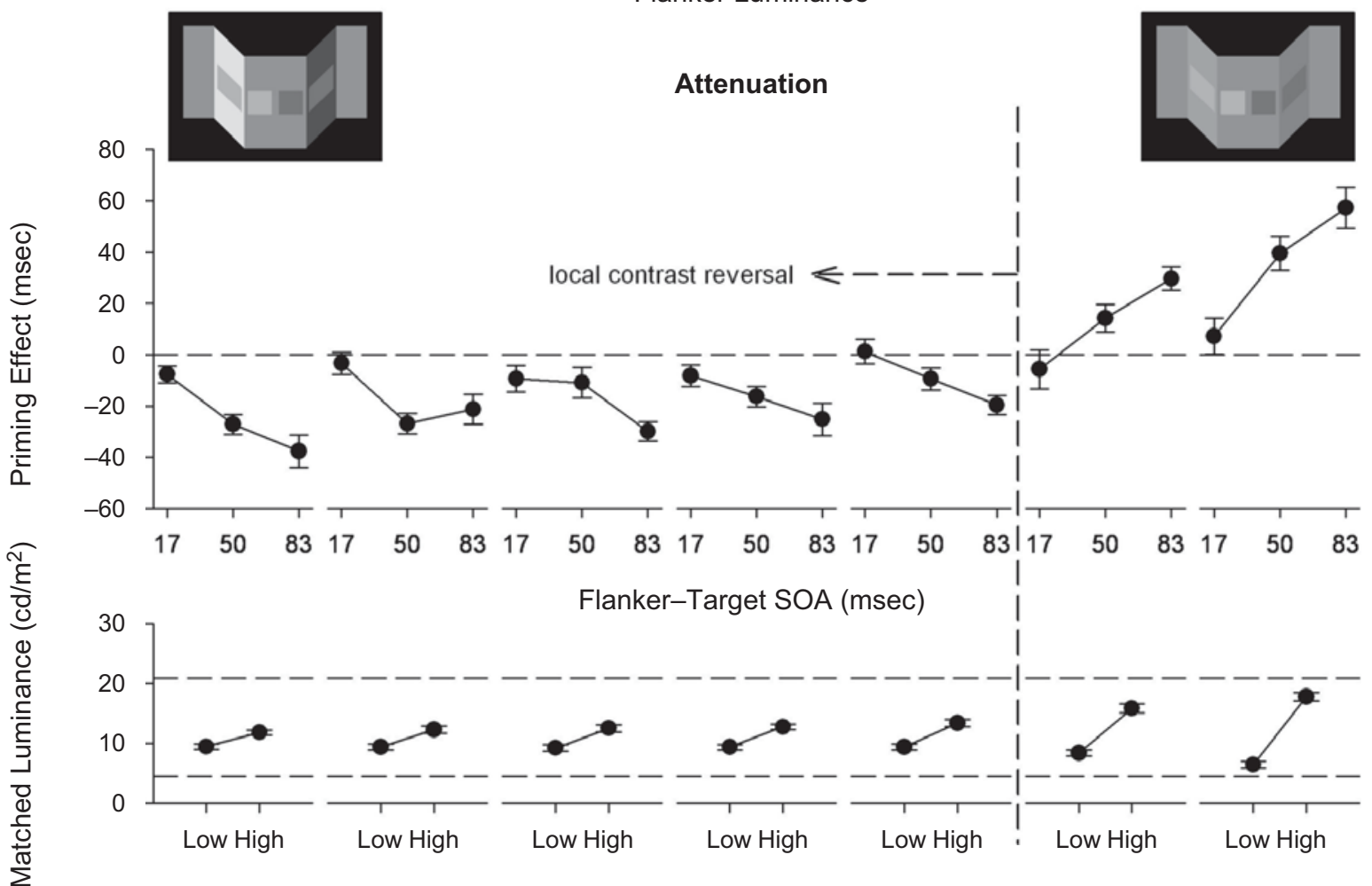

Flanker Luminance

Figure 5. Upper panels: Priming effects $\left(\mathrm{RT}_{\text {incon }}-\mathrm{RT}_{\text {con }}\right)$ and brightness matches to the adjacent flanker stimuli in the enhancement conditions in Experiment 2. Lower panels: Priming effects and brightness matches in the attenuation conditions. L:S, light:shadow ratio; SOA, stimulus onset asynchrony. Error bars represent standard errors of the means corrected for intersubject variance. 
those for the RTs: They started out positive at low lightshadow contrasts but became increasingly negative with increasing light-shadow contrast $[F(6,30)=21.1, p=$ $.0001]$. Again, an interaction of light-shadow level and SOA indicated that the absolute amplitude of the priming effect increased with SOA $[F(12,60)=5.5, p=.0086]$.

An analysis of the brightness-matching task revealed that the illusion observed in Experiment 1 was strongly modulated by changes in lighting (Figure 5). Importantly, in all the conditions, the more luminant flanker continued to appear brighter than the less luminant flanker [attenuation conditions, all $F_{\mathrm{s}}(1,5) \geq 7.4$, all $p \mathrm{~s} \leq .042$; enhancement conditions, all $F \mathrm{~s}(1,5) \geq 253.6$, all $p \mathrm{~s}<.0001]$. The perceived brightness difference between the flankers differed between light-shadow levels $[F(6,30)=26.7, p<$ $.001]$ and was judged much larger in the enhancement than in the attenuation conditions $[F(1,5)=923.2, p<.0001]$. There was a strong interaction between light-shadow level and illusion direction, such that the effect of the illusion increased with increasing contrast between the light and shadow segments $[F(6,30)=48.3, p<.0001]$.

\section{Discussion}

Experiment 2 replicates the dissociation between the priming and matching tasks observed in Experiment 1. Again, priming effects are positive under enhancement conditions but reverse under attenuation conditions, such that RTs became shorter with inconsistent flankers than with consistent flankers. At the same time, the results from the matching task show no sign of reversal in apparent brightness of the flankers: The physically more luminant flanker always appears brighter than the less luminant one, irrespective of any reversals in priming effects.

Is the reversal of the priming effect in the attenuation conditions due to a reversal in local flanker contrast? Indeed, the experiment clearly shows that priming effects reverse in exactly those conditions in which the flankers' local contrast to the background reverses, indicating that it is, indeed, local flanker-to-background contrast that drove the priming effect. As a result, the priming effect is dissociated both from the actual luminances of the flankers and from their apparent brightness values.

However, a possibility remains that the participants never responded to the brightness of the targets in the first place. Instead, they might have responded to the correct target on the basis of its local contrast sign - that is, to the target that was a local luminance increment. Under such a strategy, it would not be surprising that the local contrast of the flankers is priming responses to the local contrast of the targets. Moreover, the matching task is inappropriate, because the apparent brightness of a target would be unrelated to its local contrast sign. Instead, the appropriate matching task would be a judgment of whether a target was darker or brighter than its background, which is a trivial task that would not be dissociable from the priming effects.

Experiment 3 was designed to rule out responses to the target on the basis of local contrast sign. We employed stimuli from the attenuation and enhancement conditions in Experiment 1 but presented three different pairs of targets: both local luminance decrements (decrement targets), both luminance increments (increment targets), or one decrement and one increment (mixed targets). The participants were still instructed to respond to the brighter of the two targets. This way, the participants were forced to base their responses on a comparison of the two targetsthat is, their relative (target-to-target) contrast.

\section{EXPERIMENT 3}

\section{Method}

Participants. Six students from the University of Kaiserslautern (21-22 years of age, 2 male, 1 left-handed, all with normal or corrected-to-normal vision) participated for payment after giving informed consent.

Apparatus. The experiment was controlled by a $300-\mathrm{MHz}$ personal computer driving a 17 -in. VGA color monitor $(640 \times 480$ pixels) in synchrony with the monitor retrace rate of $60 \mathrm{~Hz}$. The monitor was viewed from a distance of approximately $80 \mathrm{~cm}$ in a dimly lit room.

Stimuli and Procedure. The experimental tasks were identical to those in the enhancement and attenuation conditions in Experiment 1 . The stimulus conditions were also identical, with the following exceptions (see Figures 6 and 7). First, we employed three possible pairs of targets: two decrement targets with lower luminance than the background $\left(1.0\right.$ and $\left.7.4 \mathrm{~cd} / \mathrm{m}^{2}\right)$, two increment targets with higher luminance than the background $\left(15.6\right.$ and $\left.34.2 \mathrm{~cd} / \mathrm{m}^{2}\right)$, or mixed targets ( 4.5 and $20.9 \mathrm{~cd} / \mathrm{m}^{2}$; same as in Experiment 1). Second, target sizes were decreased to $0.6^{\circ} \times 0.6^{\circ}$, whereas the height of the flankers was increased to make them appear roughly square-shaped. We used flanker-target SOAs of 17, 50, and $83 \mathrm{msec}$. The participants took part in four 1-h sessions, each consisting of 20 blocks of 42 trials. In a separate brightness-matching session (with the procedure identical to that in Experiments 1 and 2), the flankers and targets appeared simultaneously in all the lighting conditions, and each participant performed 4 trials per stimulus condition. In all the sessions, the stimulus conditions appeared equiprobably in pseudorandomized order.

Data treatment and statistical methods. Outlier detection in the priming task was performed as in Experiment 1. We discarded $0.35 \%$ of the data.

\section{Results}

In all the enhancement conditions, priming effects $\left(\mathrm{RT}_{\text {incon }}-\mathrm{RT}_{\text {con }}\right)$ were significantly positive [for decrement targets, $F(1,5)=34.8, p=.0020$; for mixed targets, $F(1,5)=78.7, p=.0003$; for increment targets, $F(1,5)=$ $7.1, p=.0445$; see Figures 6 and 7, upper panels]. In the attenuation conditions, however, priming effects were reversed [for decrement targets, $F(1,5)=23.0, p=.0049$; for mixed targets, $F(1,5)=33.1, p=.0022$; for increment targets, $F(1,5)=4.8, p=.0796]$, thus confirming the results of Experiments 1 and 2. In the enhancement as well as the attenuation conditions, the absolute magnitudes of the priming effects increased with $\operatorname{SOA}[F(2,10)=17.5$ and $5.27, p=.0073$ and .0312 , respectively] and were not significantly different across target pairs. Overall, RTs were about $30 \mathrm{msec}$ shorter for mixed targets than for decrement or increment targets [in enhancement conditions, $F(2,10)=12.8, p=.0101$; in attenuation conditions, $F(2,10)=24.2, p=.0011]$.

Similar effects were observed in the error rates (Figure 6, lower panel). In all the enhancement conditions, error rates were higher on inconsistent than on consistent 

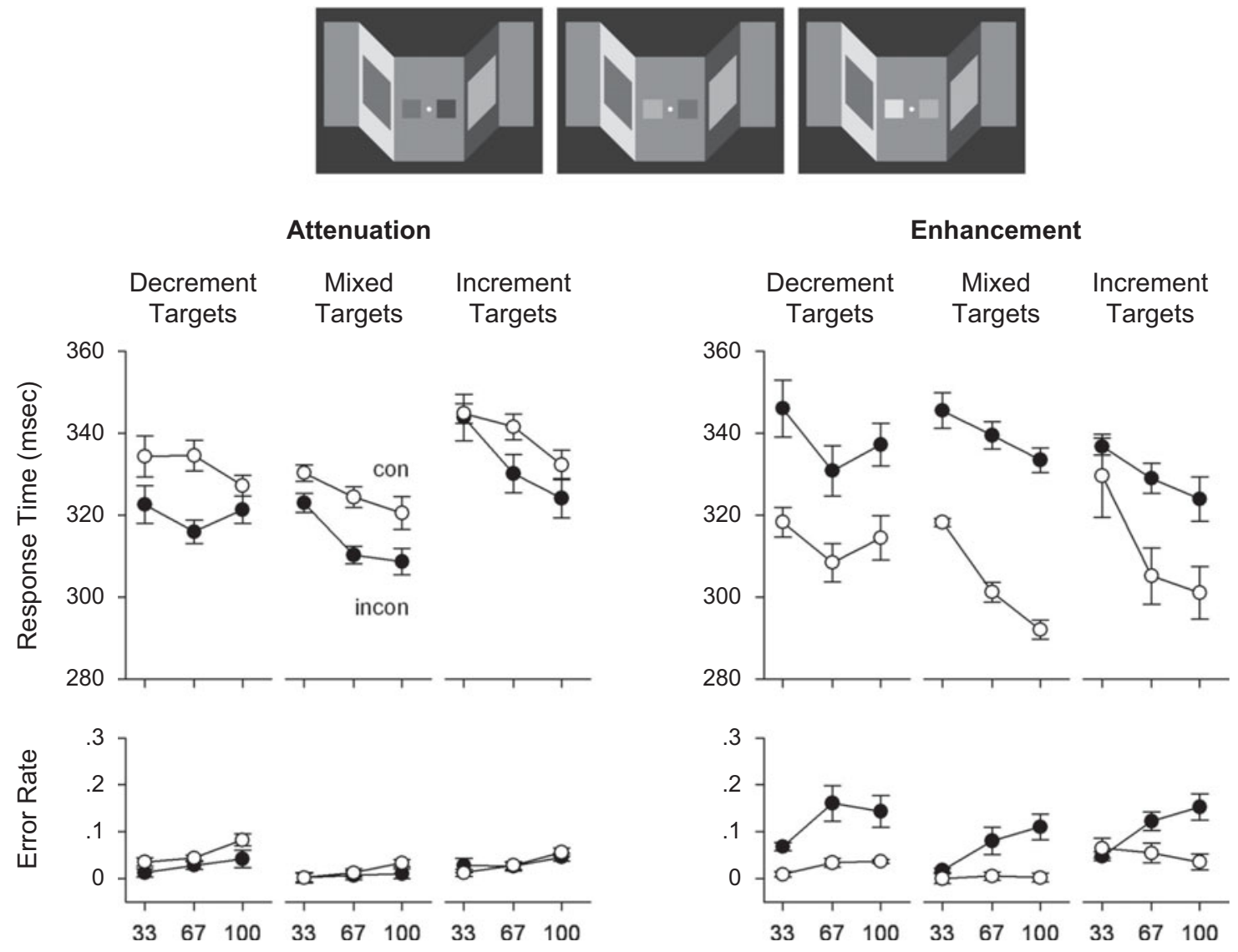

Flanker-Target SOA (msec)

Figure 6. Upper panels: Response times in Experiment 3. Lower panels: Error rates. SOA, stimulus onset asynchrony. Error bars represent standard errors of the means corrected for intersubject variance.

trials [for decrement targets, $F(1,5)=59.8, p=.0006$ for mixed targets, $F(1,5)=23.1, p=.0049$; for increment targets, $F(1,5)=2.3, p=.1900]$. In the attenuation conditions, however, the pattern was reversed, with more errors now occurring on consistent trials [for decrement targets, $F(1,5)=8.0, p=.0371$; for mixed targets, $F(1,5)=11.4$, $p=.0198$; but for increment targets, $F<1]$. In the enhancement conditions, the magnitude of this priming effect $\left(\right.$ error $_{\text {incon }}-$ error $\left._{\text {con }}\right)$ increased with SOA $[F(2,10)=$ $15.0, p=.0016]$ and also tended to do so in the attenuation conditions $[F(2,10)=4.3, p=.0526]$. Overall, error rates increased with SOA in the enhancement as well as the attenuation conditions $[F(2,10)=12.9$ and 14.6, $p=.0027$ and .0094 , respectively], and error rates were lower when the targets were mixed than when both were decrements or increments $[F(2,10)=20.4$ and $12.1, p=$ .0015 and .0039 , respectively]. Other significant effects included a main effect of target pair on priming magnitude in the enhancement as well as the attenuation conditions [both $F \mathrm{~s}(2,10)=6.5, p \mathrm{~s}=.0347$ and .0171 , respectively] and a joint effect of target pair and SOA on the priming effect in the enhancement conditions $[F(4,20)=6.3, p=$ $.0160]$, both effects indicating that priming effects were slightly more pronounced for decrement than for increment targets.

An analysis of the brightness-matching task (Figure 7, upper panel) revealed that the more luminant flanker continued to appear brighter than the less luminant flanker [attenuation condition, $F(1,5)=9.6, p=.0268$; enhancement condition, $F(1,5)=465.7, p<.0001]$. The perceived difference in brightness was much larger in the enhancement than in the attenuation conditions $[F(1,5)=$ 376.9, $p<.0001]$.

\section{Discussion}

In Experiment 3, it was impossible for the participants to respond to the target solely on the basis of local contrast sign, because the correct target could be higher as well as lower in luminance than the local background. Moreover, the correct target was no longer the one with the largest positive local contrast to the background. Instead, the target was now defined solely on the basis of relative (targetto-target) contrast. Despite these changes, Experiment 3 replicated the finding that priming effects are strongly positive in enhancement conditions but are reversed in attenuation conditions, in which the flankers switch their 

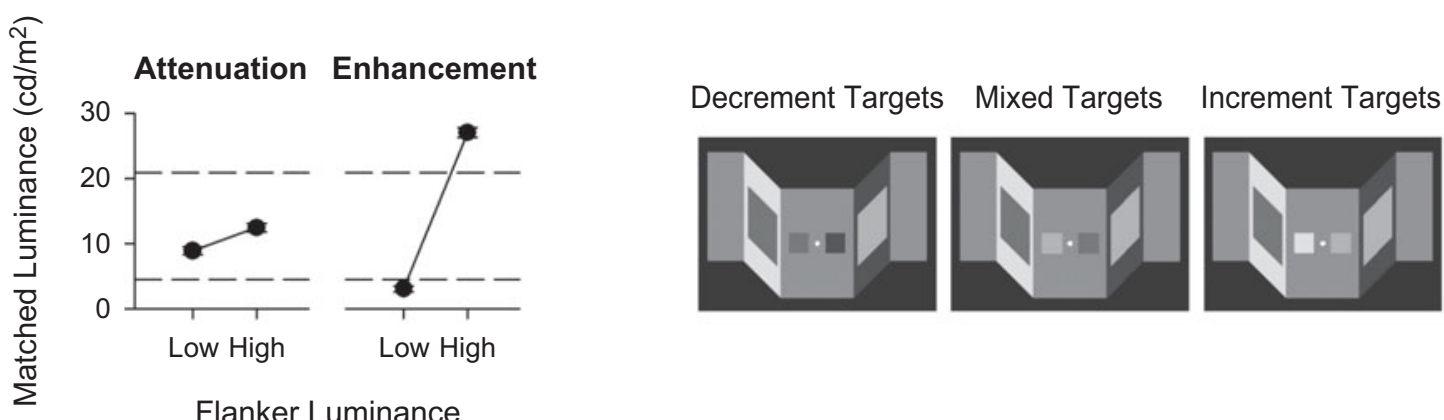

Flanker Luminance
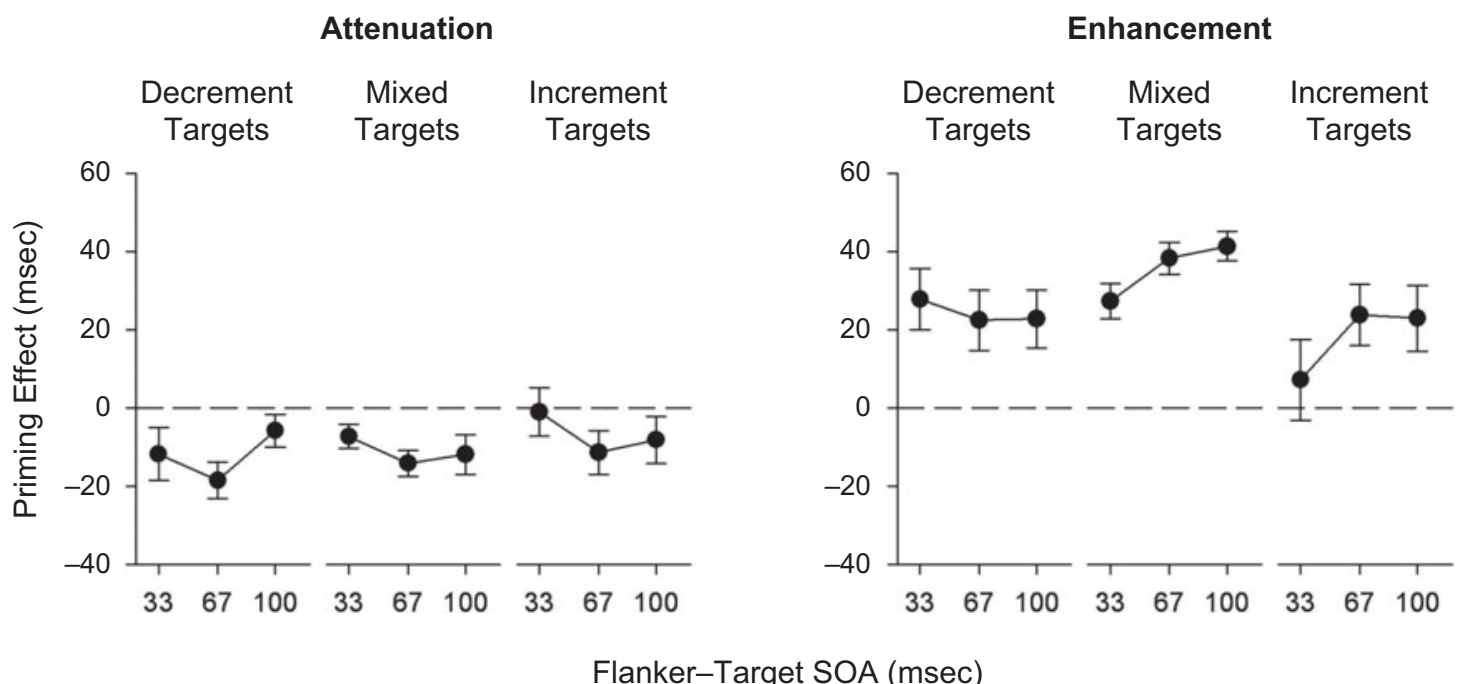

Figure 7. Upper panel: Brightness matches in Experiment 3. Lower panels: Priming effects (RT incon $_{-}$RT $\left._{\text {con }}\right)$. SOA, stimulus onset asynchrony. Error bars represent standard errors of the means corrected for intersubject variance.

local contrast sign. As in the previous experiments, the matching task revealed that the physically more luminant flanker was always judged brighter than the less luminant one in all the illusion conditions. Again, in the attenuation conditions, one flanker looked brighter than the other, yet primed as if it were darker (and vice versa). These data show that response priming effects are still determined by the local contrast of the flankers even if the correct target is defined by relative (target-to-target) contrast and that priming effects are still dissociated from the perceived brightness of the flankers, as well as from their physical luminances. ${ }^{1}$

\section{GENERAL DISCUSSION}

Response priming by luminance flankers depends neither on the actual physical luminances of the flankers nor on their subjective brightness, as assessed in a psychophysical matching task; instead, it seems to depend exclusively on the local luminance contrast of the flankers to their immediate background. As long as this local contrast is in accord with the contrast between the flankers, the more luminant flanker remains a local luminance increment, the less luminant flanker remains a local decrement, and priming effects are positive throughout. As soon as local contrast is in disagreement with the contrast between the flankers, however, the more luminant flanker turns into a local decrement (and vice versa), and priming effects are reversed. This reversal occurs in spite of the fact that in all the conditions in the present experiments, the more luminant flanker never appeared darker than the less luminant one. These findings indicate a qualitative dissociation between the subjective brightness of the stimuli and their propensity for priming a motor response: Even though one stimulus can appear brighter than another, it can trigger responses as if it were darker. Note that this dissociation makes no use of visual masking or brief stimulus presentations of any kind: It is obtained even though the presented stimuli are all in plain view, and it relies solely on characteristic differences in processing dynamics between the priming and matching tasks.

If response priming is dissociated from subjective brightness, does this mean that it is also dissociated from lightness constancy mechanisms? The illusion employed here relies on elaborate mechanisms and depends critically on the interpretation of light and shadow in the 3-D layout of the scene. Clearly, it affects both the lightness and brightness of the flankers. When the more luminant flanker appears on a shadowed segment, the visual system estimates that it must be a surface element whose reflectance is particularly high; when the same flanker appears on the lighted segment instead, the system estimates that 
its reflectance differs only a little from the background. The fact that the flankers can look either similar or dissimilar in lightness and brightness, depending on the illusion condition, is a consequence of this process of taking account of illumination and spatial layout. Therefore, the illusion employed here is a direct consequence of lightness constancy. If priming effects are in systematic disagreement with the flankers' perceived brightness values, this suggests that at least some aspects of lightness constancy are absent from the response activation process. ${ }^{2}$

Our conclusions are in agreement with a study by Moore and Brown (2001), in which visual search for highluminance and low-luminance targets was performed under conditions in which parts of the display seemed to be watched through a dark transparent filter. The search target was always defined by its lightness (i.e., perceived reflectance), irrespective of the filtering. The authors found that when lightness information conflicted with luminance information (e.g., when a high-luminance target viewed through a dark filter had the same luminance as the distractors outside the filter), visual search was slower than when lightness and luminance information were in agreement (e.g., when a high-luminance target appeared outside the filter and was thus the single most luminant item in the display). These results suggest that visual search is not entirely based on lightness information but is also influenced by raw luminance. The authors concluded that visual search had been initiated before scene processing and lightness constancy were complete.

This conclusion might seem to conflict with some physiological studies showing that subjective lightness is signaled early in the processing hierarchy, such that the responses of many cells in the primary visual cortex (V1) and even some in the lateral geniculate nucleus are modulated by a simultaneous contrast illusion (MacEvoy \& Paradiso, 2001; Rossi \& Paradiso, 1999; Rossi, Rittenhouse, \& Paradiso, 1996). However, several things should be noted here. First, there is also a substantial number of cells in the recorded areas that do not respond to simultaneous contrast. Second, in the studies above a method was used in which simultaneous contrast was modulated sinusoidally over time and entrainment of neuronal responses to the local contrast level was monitored. This method is elegant but obscures any information as to how soon the neurons' responses become responsive to the illusion; perhaps they are modulated by recurrent signals from cells further downstream the processing hierarchy. Third, simultaneous contrast is not a clear example of lightness constancy, because it appears in the absence of clear illumination or transparency cues (see Gilchrist, 2006, for the numerous puzzles surrounding simultaneous contrast). In contrast to that, the transparency stimuli employed by Moore and Brown (2001) require the visual system to correctly assign luminance edges to filtered and unfiltered regions of the display. Single-cell recordings in visual area V2 show that edge assignments in transparent stimuli are signaled only after a delay of 50-100 msec, during which the cells signal local luminance contrast only (Qiu \& von der Heydt, 2007). This seems to leave a comfortable time window in which to pass preconstancy contrast information on through the visuomotor system.

Breitmeyer, Ro, and Singhal (2004; Breitmeyer, Öğmen, \& Chen, 2004) have proposed that response priming is based on raw physical stimulus characteristics, such as the distribution of wavelength information, instead of perceived color. We adopt the less radical view that response priming reflects a stimulus representation that is earlier and less elaborate than the one underlying conscious vision and, consequently, deficient in lightness constancy. This idea is in line with current models of lightness perception (Adelson, 2000; Gilchrist et al., 1999), which assume that a globally integrated interpretation of the visual scene is obtained from an earlier representation of strictly local image information that is gradually processed to incorporate information about lighting, transparency, and 3-D layout. In the framework of the rapid-chase model of response priming (Schmidt et al., 2006; Schmidt \& Schmidt, 2009), this view suggests that the feedforward sweeps successively triggered by the flankers and targets are still dominated by local contrast signals. The conscious perception of lightness and brightness will be determined later in processing, probably as the result of recurrent interactions trailing behind the initial processing wave (Lamme \& Roelfsema, 2000; see also Di Lollo et al., 2000). Of course, this idea is consistent with other dissociations between response priming and awareness, such as the independence of priming from visual backward masking (Mattler, 2003b; Vorberg et al., 2003).

The dissociation between brightness perception and response priming observed here indicates that the two measures are based on qualitatively different stimulus representations (Schmidt \& Vorberg, 2006). Whereas the lightness-constant representation in visual awareness results from trying to look "behind" the raw contrast image to arrive at a valid reconstruction of the actual surface properties, the representation underlying rapid response activation might in a way be "less than meets the eye," or at least less than meets visual awareness. Maybe this finding points toward a more fundamental distinction between fast motor measures of perception (such as speeded keypress or pointing responses), which seem largely based on rapid feedforward activation, and traditional psychophysical measures, which typically leave the observer enough time to report the final outcome of processing in visual awareness. In contrast to such "slow psychophysics," visuomotor measures of perception could be regarded as "fast psychophysics" that might be able to capture early, preconscious processing stages otherwise inaccessible to conscious report.

\section{AUTHOR NOTE}

This work was supported by the German Research Foundation, Grant Schm1671/1 to T.S. We thank Karl Gegenfurtner, Filipp Schmidt, Anke Haberkamp, and Anna Seydell for valuable discussions. Correspondence concerning this article should be addressed to T. Schmidt, Faculty of Social Sciences, Psychology I, University of Kaiserslautern, ErwinSchrödinger-Str. Geb. 57, D-67663 Kaiserslautern, Germany (e-mail: thomas.schmidt@sowi.uni-kl.de). 


\section{REFERENCES}

Adelson, E. H. (1993). Perceptual organization and the judgment of brightness. Science, 262, 2042-2044.

AdELSON, E. H. (2000). Lightness perception and lightness illusions. In M. S. Gazzaniga (Ed.), The new cognitive neurosciences (2nd ed., pp. 339-351). Cambridge, MA: MIT Press.

Bakeman, R., \& McArthur, D. (1996). Picturing repeated measures: Comments on Loftus, Morrison, and others. Behavior Research Methods, Instruments, \& Computers, 28, 584-589.

Breitmeyer, B. G., ÖĞMen, H., \& Chen, J. (2004). Unconscious priming by color and form: Different processes and levels. Consciousness \& Cognition, 13, 138-157.

Breitmeyer, B. G., Ro, T., \& Singhal, N. S. (2004). Unconscious color priming occurs at stimulus- not percept-dependent levels of processing. Psychological Science, 15, 198-202.

Bullier, J. (2001). Integrated model of visual processing. Brain Research Reviews, 36, 96-107.

Dehaene, S., Naccache, L., Le Clec'H, G., Koechlin, E., MuelLer, M., Dehaene-Lambertz, G., et AL. (1998). Imaging unconscious semantic priming. Nature, 395, 597-600.

Di Lollo, V., Enns, J. T., \& Rensink, R. A. (2000). Competition for consciousness among visual events: The psychophysics of reentrant visual processes. Journal of Experimental Psychology: General, 129, 481-507.

Eimer, M., \& Schlaghecken, F. (1998). Effects of masked stimuli on motor activation: Behavioral and electrophysiological evidence. Journal of Experimental Psychology: Human Perception \& Performance, 24, 1737-1747.

Eimer, M., \& Schlaghecken, F. (2003). Response facilitation and inhibition in subliminal priming. Biological Psychology, 64, 7-26.

Eriksen, B. A., \& EriKsen, C. W. (1974). Effects of noise letters upon the identification of a target letter in a nonsearch task. Perception \& Psychophysics, 16, 143-149.

GiLchrist, A. (2006). Seeing black and white. Oxford: Oxford University Press.

Gilchrist, A., Kossyfidis, C., Bonato, F., Agostini, T., CataLIOTTI, J., LI, X., ET AL. (1999). An anchoring theory of lightness perception. Psychological Review, 106, 795-834.

JAŚKowski, P., VAn der Lubbe, R. H. J., Schlotterbeck, E., \& VerLEGER, R. (2002). Traces left on visual selective attention by stimuli that are not consciously identified. Psychological Science, 13, 48-54.

Lamme, V. A. F., \& Roelfsema, P. R. (2000). The distinct modes of vision offered by feedforward and recurrent processing. Trends in Neurosciences, 23, 571-579.

LAND, E. H., \& MCCANn, J. J. (1971). Lightness and retinex theory. Journal of the Optical Society of America, 61, 1-11.

Leuthold, H., \& Kopp, B. (1998). Mechanisms of priming by masked stimuli: Inferences from event-related brain potentials. Psychological Science, 9, 263-269.

LI, X., \& GILCHRIST, A. L. (1999). Relative area and relative luminance combine to anchor surface lightness values. Perception \& Psychophysics, 61, 771-785.

Loftus, G. R., \& Masson, M. E. J. (1994). Using confidence intervals in within-subject designs. Psychonomic Bulletin \& Review, 1, 476-490.

MacEvoy, S. P., \& Paradiso, M. A. (2001). Lightness constancy in primary visual cortex. Proceedings of the National Academy of Sciences, 98, 8827-8831.

Mattler, U. (2003a). Delayed flanker effects on lateralized readiness potentials. Experimental Brain Research, 151, 272-288.

Mattler, U. (2003b). Priming of mental operations by masked stimuli. Perception \& Psychophysics, 65, 167-187.

Moore, C. M., \& Brown, L. E. (2001). Preconstancy information can influence visual search: The case of lightness constancy. Journal of Experimental Psychology: Human Perception \& Performance, 27, 178-194.

Neumann, O. (1990). Direct parameter specification and the concept of perception. Psychological Research, 52, 207-215.
Neumann, O., \& Klotz, W. (1994). Motor responses to nonreportable, masked stimuli: Where is the limit of direct parameter specification? In C. Umiltà \& M. Moscovitch (Eds.), Attention and performance XV: Conscious and nonconscious information processing (pp. 123-150). Cambridge, MA: MIT Press.

QIU, F. T., \& VON DER HEYDT, R. (2007). Neural representation of transparent overlay. Nature Neuroscience, 10, 283-284.

Rossi, A. F., \& Paradiso, M. A. (1999). Neural correlates of perceived brightness in the retina, lateral geniculate nucleus, and striate cortex. Journal of Neuroscience, 19, 6145-6156.

Rossi, A. F., Rittenhouse, C. D., \& Paradiso, M. A. (1996). The representation of brightness in primary visual cortex. Science, 273, 1104-1107.

SchmidT, T. (2002). The finger in flight: Real-time motor control by visually masked color stimuli. Psychological Science, 13, 112-118.

Schmidt, T., Niehaus, S., \& Nagel, A. (2006). Primes and targets in rapid chases: Tracing sequential waves of motor activation. Behavioral Neuroscience, 120, 1005-1016.

Schmidt, T., \& Schmidt, F. (2009). Processing of natural images is feedforward: A simple behavioral test. Attention, Perception, \& Psychophysics, 71, 594-606.

Schmidt, T., \& Vorberg, D. (2006). Criteria for unconscious cognition: Three types of dissociation. Perception \& Psychophysics, 68 489-504.

VAnRullen, R., \& Thorpe, S. J. (2002). Surfing a spike wave down the ventral stream. Vision Research, 42, 2593-2615.

VAth, N., \& Schmidt, T. (2007). Tracing sequential waves of rapid visuomotor activation in lateralized readiness potentials. Neuroscience, 145, 197-208.

Vorberg, D., Mattler, U., Heinecke, A., Schmidt, T., \& SchwarzBACH, J. (2003). Different time courses for visual perception and action priming. Proceedings of the National Academy of Sciences, $\mathbf{1 0 0}$, 6275-6280.

\section{NOTES}

1. In the RT task, the presentation times for the flankers and targets were limited by the time needed to respond to the targets, whereas viewing time was unlimited in the matching task. Thus, it could be argued that the illusion might look different or even be reversed at shorter presentation times, so that it might finally be in agreement with the direction of the priming effects. As a control, we repeated the brightness-matching task of Experiment 3 with two experienced observers (the last two authors) under conditions in which the stimulus kept flashing up for $300 \mathrm{msec}$ (about the average RT in the priming task), 200, 100, or $50 \mathrm{msec}$ at interflash intervals of $750 \mathrm{msec}$, while participants continued to look at the fixation point. The matching results were virtually unaffected by presentation duration, even for presentations as short as $50 \mathrm{msec}$.

2. Differentiation of the terms lightness and brightness is intricate and can be misleading. Although their formal definition as perceived reflectance and perceived luminance is straightforward enough, practical aspects of measurement render the concepts more ambiguous. For example, a stimulus depicted on a monitor screen can be judged in the pictorial reference system (e.g., as part of the folded plane depicted in our experiments) or in a real-world reference system (e.g., as part of the monitor surface). A lightness judgment would evaluate a part of the pictured surface in the pictorial world, whereas a brightness judgment would evaluate a part of the monitor surface in the real world. Gilchrist (2006) stressed the important role of alternative spatial reference systems in the interpretation of lightness. It is ironic that the same author dismissed models of brightness perception (and, indeed, the entire notion of brightness constancy) as relatively unimportant for understanding lightness perception, when shifts in spatial reference systems are frequently the only thing differentiating lightness from brightness judgments.

(Manuscript received July 24, 2008; revision accepted for publication March 22, 2010.) 